\title{
Advanced Magnetic Force Microscopy Tips for Domain Images of Soft Magnetic Materials under Magnetic Field
}

\author{
S. H. Liou
}

Department of Physics and Astronomy and Center for Materials Research and Analysis, University of Nebraska, Lincoln, NE 68588-0111

We have developed low stray field and high coercivity magnetic force microscopy (MFM) tips for the domain images of soft magnetic materials under magnetic field. A systematic domain evolution under magnetic field can clearly be observed by MFM in a garnet sample.

Magnetic domain structures of garnets have been studied by ferrofluid decoration methods and magneto-optic Faraday effect etc.[1,2] The resolutions of these methods are limited by either the size of the magnetic particles used in the ferrofluid or the wavelength of the light used in the magneto-optic Faraday effect. The recently developed magnetic force microscopy has been proved to have a higher resolution than ferrofluid decoration methods and magneto-optic Faraday effect in magnetic domain imaging. There are a few investigations of the magnetic domain structures of garnets with or without external magnetic field using the MFM method. However, imaging magnetic domains of soft magnetic materials (low magnetic coercive field materials such as garnets) needs some special precautions due to the fact that the domain structures of soft magnetic materials are easily perturbed by the stray field from the magnetic force microscopy tips [3]. It is also noted that many studies use soft tips. The soft MFM tip is more difficult for magnetic domain images under magnetic field, due to the change of magnetization direction of the tip under magnetic field. The ideal magnetic force microscopy tip for imaging magnetic domains of soft magnetic materials should have a high coercive and low stray field.

In this paper, we have systematically studied magnetic domains of an epitaxial garnet film using magnetic force microscopy. The magnetic garnet is an epitaxial YGdTmGa/YSmTmGa film, grown on a nonmagnetic $\mathrm{Ga}_{3} \mathrm{Ga}_{5} \mathrm{O}_{12}$ (GGG) substrate, with a zero-field stripe width $1.4 \mu \mathrm{m}$. To our knowledge, we have fabricated CoPt MFM tips with low stray field and high $\mathrm{H}_{\mathrm{c}}$ for the first time. We demonstrated that these MFM tips can be used in the study of magnetic domain images of soft magnetic materials in the presence of an external magnetic field. An example of this type study is shown in Fig.1. In this case, the magnetic field was applied along the easy axis (perpendicular to the film surface). The magnetization curve was measured by a SQUID magnetometer, as shown in Fig. 2. That the domain structure of the garnet is sequentially varied with the applied magnetic field is clearly observed, showing that we are able to directly correlate the magnetic domain patterns with the magnetization curve. The MFM tip does not appear to disturb the domain structure of the garnet. The new developed MFM tips show great promise for magnetic domain images of soft magnetic materials under magnetic field. We show the direct correlation of the domain structure and the magnetic hystersis curve. The moving of the domain wall structure that is affected by the stray field emanating from the tip is only noted in the high magnetic field range from 190 Oe to 290 Oe. In this field range the magnetic domain wall is easy to move. The MFM tip has been cycled through 
the positive and negative magnetic field. There are not any noticeable effects on the change of the magnetization direction of the MFM tip under the external magnetic field.

\section{References}

1. R. R. Katti, P. Rice, J. C. Wu, H. L. Stadler, IEEE Trans. Magn. 28, (1992) 2913.

2. A. Wadas, J. Moreland, P. Rice, R.R. Katti, Appl. Phys. Lett. 64, (1994) 156.

3. Fang Tian, Chen Wang, Guangyi Shang, Naixin Wang, Chunli Bai, J. Magn. Magn. Mater. 171, (1997) 135.

4. Research was supported by the Army Research Office under grant number DAAD1900-1-0119 and Nebraska Research Initiative at the University of Nebraska.

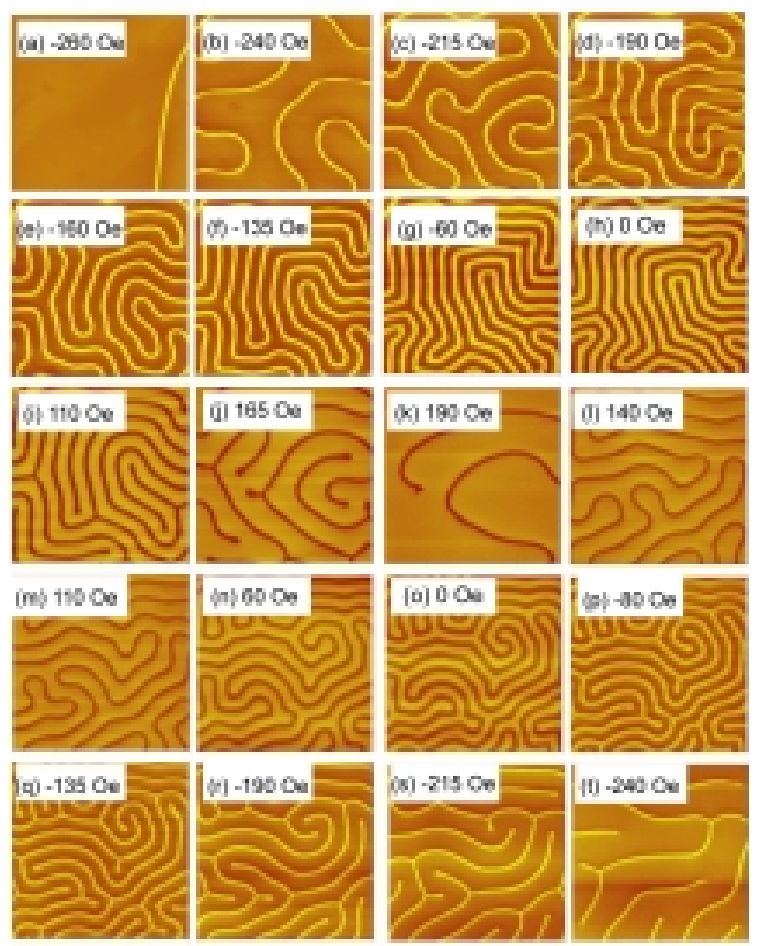

$20 \mu \mathrm{m}$

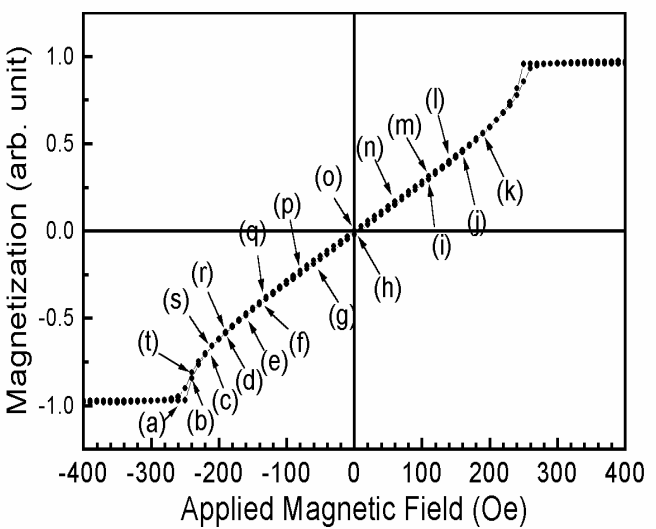

Fig. 2 The magnetization curve of a garnet measured with the field perpendicular to the film surface. The labels correspond to these in Fig.1.

Fig 1 Magnetic domain images of a garnet film under magnetic field. 